\title{
NEW MEMBERS OF THE TW HYDRAE ASSOCIATION, $\beta$ PICTORIS MOVING GROUP, AND TUCANA/HOROLOGIUM ASSOCIATION
}

\author{
INSEOK SONG ${ }^{1}$ AND B. ZUCKERMAN \\ NASA Astrobiology Institute and Department of Physics and Astronomy, University of California, \\ Los Angeles, Los Angeles, CA 90095 \\ AND \\ M. S. BESSELL \\ Research School of Astronomy and Astrophysics, Institute of Advanced Studies, \\ The Australian National University, ACT 2611, Australia \\ Received 2003 June 20; accepted 2003 August 19
}

\begin{abstract}
We have identified five new members of the TW Hydrae association (TWA), 11 new members of the $\beta$ Pic moving group, and 11 new Tucana/Horologium association members. These are the three youngest $(\$ 30$ Myr) known kinematic stellar groups near the Earth. Newly identified $\beta$ Pic group members are located mostly in the northern hemisphere, and they have a slightly different $U$-component of Galactic velocity compared to that of previously known members. Tracing the motion of $\beta$ Pic members backward in time for $12 \mathrm{Myr}$ indicates that they might have formed in a small region with an initial velocity dispersion of $\sim 8 \mathrm{~km}$ $\mathrm{s}^{-1}$. A couple of mid-M spectral type $\beta$ Pic members show emission features [He I $\lambda 5876+\lambda 6678$ ) and $\mathrm{Na} \mathrm{D}$ $\lambda 5890+\lambda 5896)$ ] seen among earlier spectral type stars in the TWA and $\beta$ Pic groups. To derive the distances of the non-Hipparcos members of these groups, we have constructed a $V-K$ versus $M_{K}$ color-magnitude diagram that is very useful in separating young $\mathrm{K} / \mathrm{M}$ stars from older main-sequence counterparts and constraining theoretical pre-main-sequence evolutionary tracks. All newly identified K- and M-type members of the three groups show saturated X-ray activity $\left(L_{\mathrm{X}} / L_{\mathrm{bol}} \sim 10^{-3}\right)$. One newly identified TWA member, SSS $101727-5354$, is estimated to be only 22 pc away from Earth. Its extreme youth, late spectral type ( M5), and proximity to Earth make SSS 101727-5354 perhaps the best target for direct imaging detection of cooling planets.
\end{abstract}

Subject headings: open clusters and associations: individual (TW Hydrae, $\beta$ Pictoris, Tucana) stars: evolution — stars: kinematics — stars: pre-main-sequence

\section{INTRODUCTION}

The past few years have seen the discovery of numerous groups of nearby young stars (Jayawardhana \& Greene 2001; Zuckerman et al. 2001a and references therein). Among these young stellar groups, the TW Hydrae association (TWA; Kastner et al. 1997; de la Reza et al. 1989; Webb et al. 1999; Zuckerman et al. 2001c), $\beta$ Pic moving group (Barrado y Navascués et al. 1999; Zuckerman et al. 2001a), and Tucana/Horologium association (Tucana/HorA; Zuckerman \& Webb 2000; Torres et al. 2000; Zuckerman, Song, \& Webb 2001b) are the most prominent. Each of these three groups can be distinguished from the other two by means of location in space, Galactic space motion $(U V W)$, and age. Their ages (8-30 Myr) and distances from Earth $(\$ 60 \mathrm{pc})$ make members of these groups the prime targets of direct imaging searches for cooling planets and circumstellar dusty disks. In fact, many members of these groups do show substellar companions (e.g., TWA 5 and HR 7329) or prominent protoplanetary/debris disks (e.g., $\beta$ Pic, TW Hya, HR 4796A, and HD 98800). Since the age range of these groups overlaps very important epochs of planet formation in our solar system (gas giant planet formation in $\lesssim 10 \mathrm{Myr}$ and terrestrial planet formation in $\lesssim 30$ Myr; ${ }^{2}$ Jacobson 2003), any planets and/or disks to be found

\footnotetext{
${ }^{1}$ UCLA Center for Astrobiology Research Scientist.

2 An upper limit timescale for Earth's core formation has been determined from chronometry using the Hafnium-Tungsten system.
}

around these stars will provide crucial information on formation and early evolution of planetary systems.

The importance of the nearby young stellar groups and their members is manifested by the fact that all members of the TWA and $\beta$ Pic groups have been selected as targets in various space- and ground-based observational programs soon after their identification. In this paper, we present newly identified members of the TWA, $\beta$ Pic, and Tucana/ HorA groups.

\section{OBSERVATIONS AND DATA ANALYSIS}

At Siding Spring Observatory (SSO; Australia) and Lick Observatory (USA), we are in the midst of an extensive survey to search for young stars near to Earth (I. Song, M. S. Bessell, \& B. Zuckerman 2004, in preparation). We have been using the double-beam grating spectrograph (DBS) and the echelle spectrograph on the two Nasmyth foci of the Australian National University's $2.3 \mathrm{~m}$ telescope.

The red channel of the DBS covers the spectral range $6500-7450 \AA$ at a measured resolution of $1.16 \AA(0.55 \AA$ pixel $\left.^{-1}\right)$. Eight orders of the echelle spectra cover portions of the wavelength range from 5800 to $7230 \AA$. We have focused on orders that contain the $\mathrm{H} \alpha$ and $\mathrm{Li} \lambda 6708$ lines. In these orders, the measured resolution was $0.45 \AA(0.17 \AA$ pixel $\left.^{-1}\right)$. Radial velocities were determined by crosscorrelating target and radial velocity standard spectra over 5 or 6 orders of the echelle, which were chosen to produce strong correlations and have few atmospheric features. 


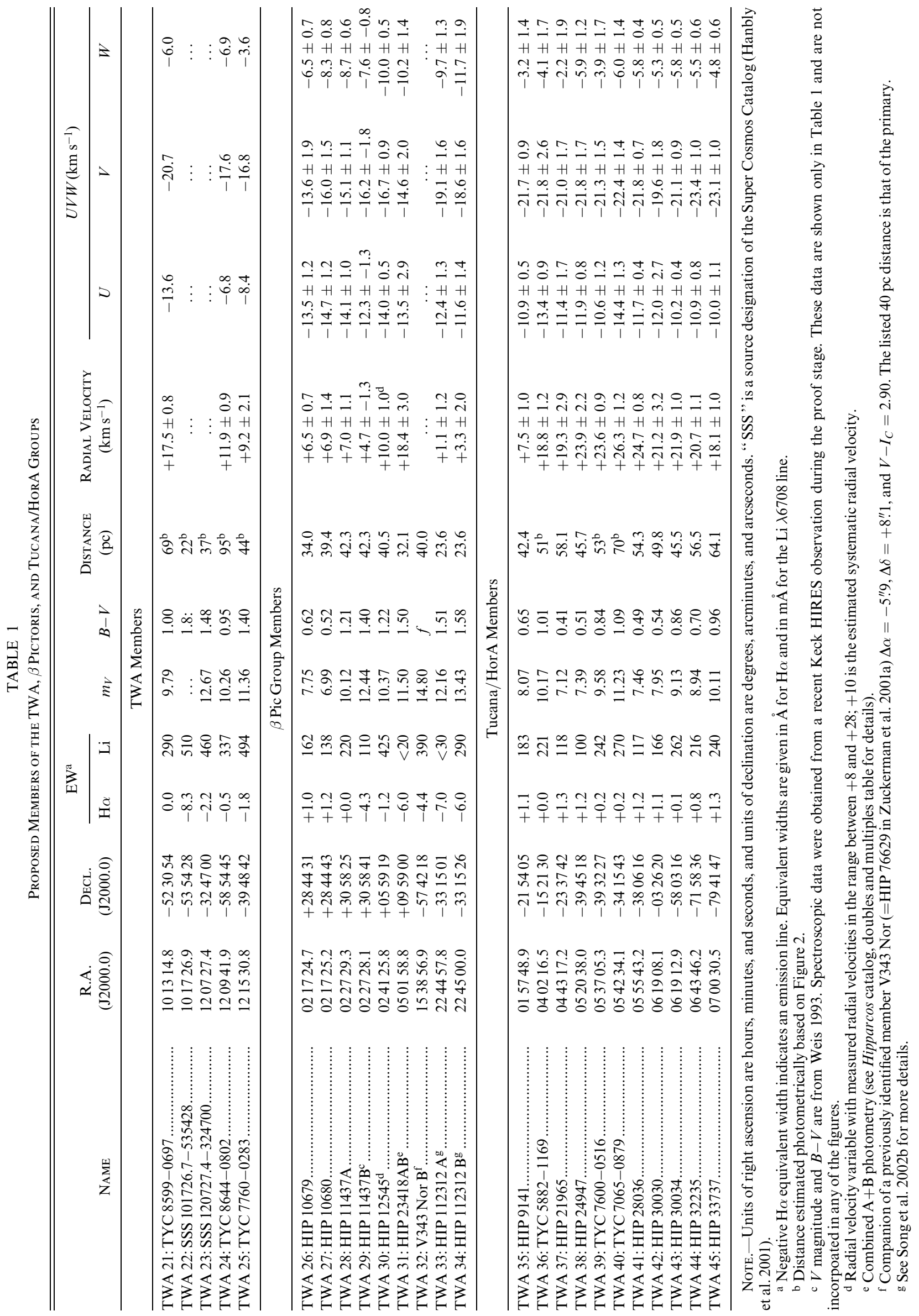


Equivalent widths of $\mathrm{H} \alpha$ and $\mathrm{Li} \lambda 6708$ were measured using an IRAF task splot.

It is an interesting coincidence that the observations of the $\beta$ Pic moving group were made with a telescope on Siding Spring Mountain that was itself formed by basaltic lava flows from the Warrumbungle volcano at roughly the same time (14-17 Myr ago; Duggan \& Knutson1991) that the stars formed.

Throughout this study, we have used a "right-handed" coordinate system for Galactic velocities: $U$ is positive toward the Galactic center, $V$ is positive in the direction of the Galactic rotation, and $W$ is positive toward the north Galactic pole.

\subsection{New Members of the $\beta$ Pictoris Moving Group}

We have identified five binary Hipparcos stars as likely $\beta$ Pic moving group members (Table 1 and Fig. 1). In addition, we identified a companion of a previously reported $\beta$ Pic member, V343 Nor.

$B V I_{c}$ photometric data were obtained for HIP 12545 , 23418, and $112312 \mathrm{~A}$ and $\mathrm{B}$ with the $0.9 \mathrm{~m}$ telescope at SSO as part of a large photometry program (R. Shobbrook et al. 2004, in preparation). From these photometric data, 2MASS data, and data from the literature, we plot the new and previously identified $\beta$ Pic members on a $V-K$ colormagnitude diagram (CMD; Fig. 2). A $V-K$ versus $M_{K}$ $\mathrm{CMD}$ is quite useful because photometric measurement errors, any uncertainties in zero-points or passband place-

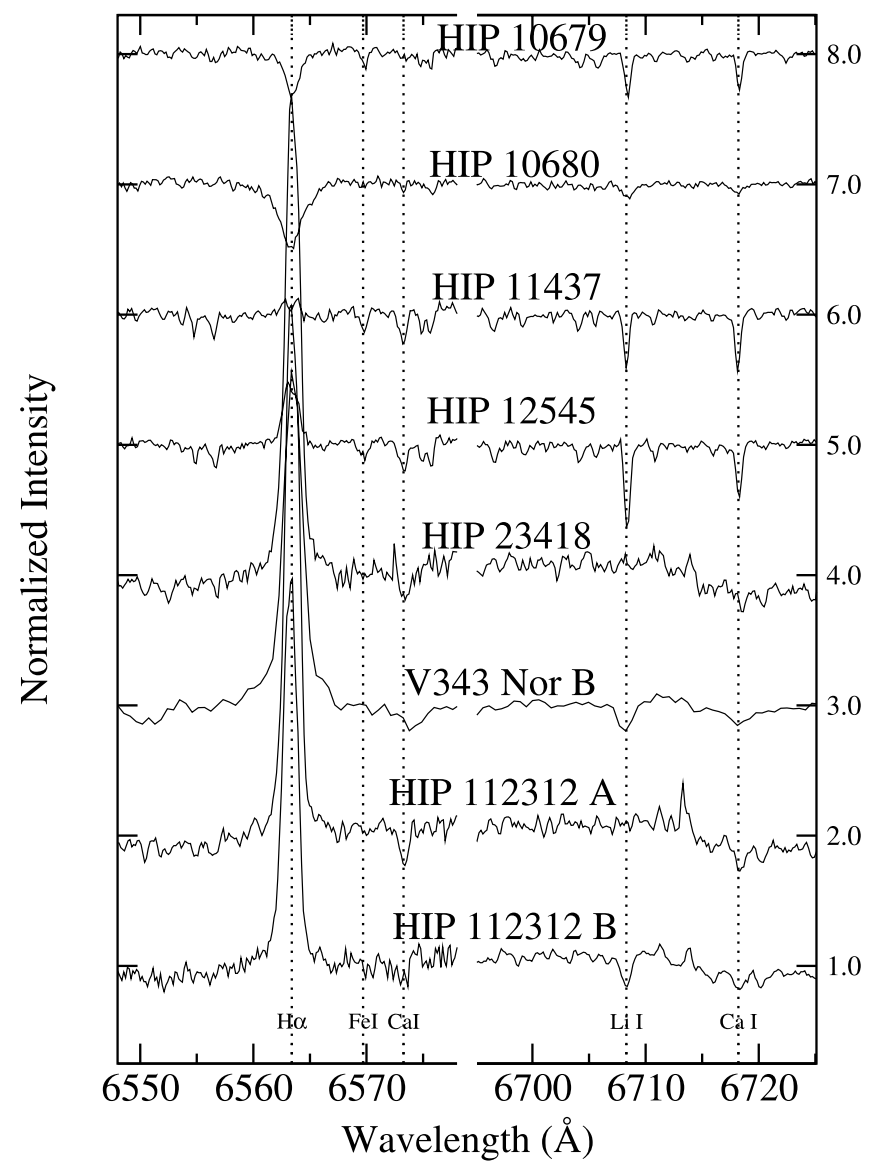

FIG. 1.-Echelle spectra of newly identified $\beta$ Pic members except DBS spectrum shown for V343 Nor B. HIP 12545 is a radial velocity variable and, thus, likely a spectroscopic binary. ments, and intrinsic stellar variability have less effect thanks to a larger baseline $(V-K)$ compared to that of other colors (e.g., $B-V, V-I_{C}$, and $\left.J-H\right)$. Cohen, Persson, \& Frogel (1978) pointed out the great advantages in $V-K$ for globular cluster giants, for instance, in the mid-1970s. However, a $V-K \mathrm{CMD}$ has seldom been used in young star studies because of the relative lack of theoretical model $V-K$ colors and the not commonly available $K$-band photometry data. We compared four solar metallicity pre-main-sequence theoretical evolutionary tracks (Baraffe et al. 1998; D’Antona \& Mazzitelli 1997; Palla \& Stahler 1993; Siess, Dufour, \& Forestini 2000) against a set of $\sim 10$ Myr old stars (Fig. 2). Model effective temperatures ( $\left.T_{\text {eff }}\right)$ and luminosities $(L)$ were converted to $V-K$ and $M_{K}$ using a relation among $T_{\text {eff }}$, broadband colors, and bolometric correction suggested by Kenyon \& Hartmann (1995).

Figure 2 suggests the following:

1. When a common transformation between stellar luminosity and temperature and observed $V$ - and $K$-band brightness is used, then the evolutionary tracks from the four models agree well except at young ages for G- and K-type stars

2. At $100 \mathrm{Myr}$, the theoretical tracks underpredict the $K$-band brightness of late $\mathrm{K}$ - and early $\mathrm{M}$-type stars

Part of the discrepancy between model predictions and empirical isochrones of old stars later than $\sim \mathrm{M} 4$ may be due to the fact that the observed Gliese stars statistically are $\gg 100$ Myr old, whereas the models are for stars that are 100 Myr old, and mid-M and later spectral type stars may not have yet settled onto the main sequence in $100 \mathrm{Myr}$. The same trend of models being brighter than the observations may also be true for very late M-type $\sim 10$ Myr old stars (see Fig. 2). If so, the discrepancy may be due to some missing opacity sources among late-type stars in theoretical evolutionary models. We note from Figure 2 that when the $T_{\text {eff- }}$ color transformation table of Siess, Forestini, \& Dougados (1997) is used to generate $V-K$ versus $M_{K}$ data for the Siess et al. (2000) model, the discrepancy between the model and observed trends is very severe $(\sim 2.5 \mathrm{mag})$. Also for a given set of coeval stars, the model predicts no change in $M_{K}$ between $V-K=4.0$ and 6.0 (M1-M5), which is physically unreasonable. Since this behavior is not seen on a $V-I_{C}$ versus $M_{V}$ CMD with the Siess et al. (2000) model data and the Siess et al. (1997) color transformation, or with the same data on a $V-K$ versus $M_{K}$ CMD with Kenyon \& Hartmann (1995) color table, it implies a flaw in the Siess et al. (1997) $K$ color to effective temperature transformation table.

Because of the well-defined location of $\sim 10$ Myr old stars in Figure 2, one can derive a reliable photometric distance to a $\sim 10$ Myr old star. For example, the distance to TYC 8599-0697, a new TWA member, was derived from visual and $K$-band magnitudes ( $V=9.79$ and $K=7.19$ ) by placing it on the observational $10 \mathrm{Myr}$ isochrone in Figure 2. From $V-K=2.60$, an absolute $K$ magnitude of $\sim 3.0$ was estimated for TYC 8599-0697, from which we calculated a distance of 69 pc. Similarly, a distance of 22 pc to SSS 101727-5354 was derived by using a spectral type M5 (which is estimated from its TiO5 index of 0.29) and, from Figure 2, an absolute $K$ magnitude of 6.0. This remarkably nearby distance (see Tables 1 and 2 for distances to all proposed TWA members) is also suggested by the very large

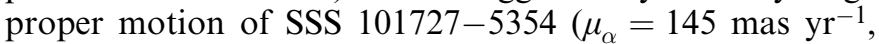




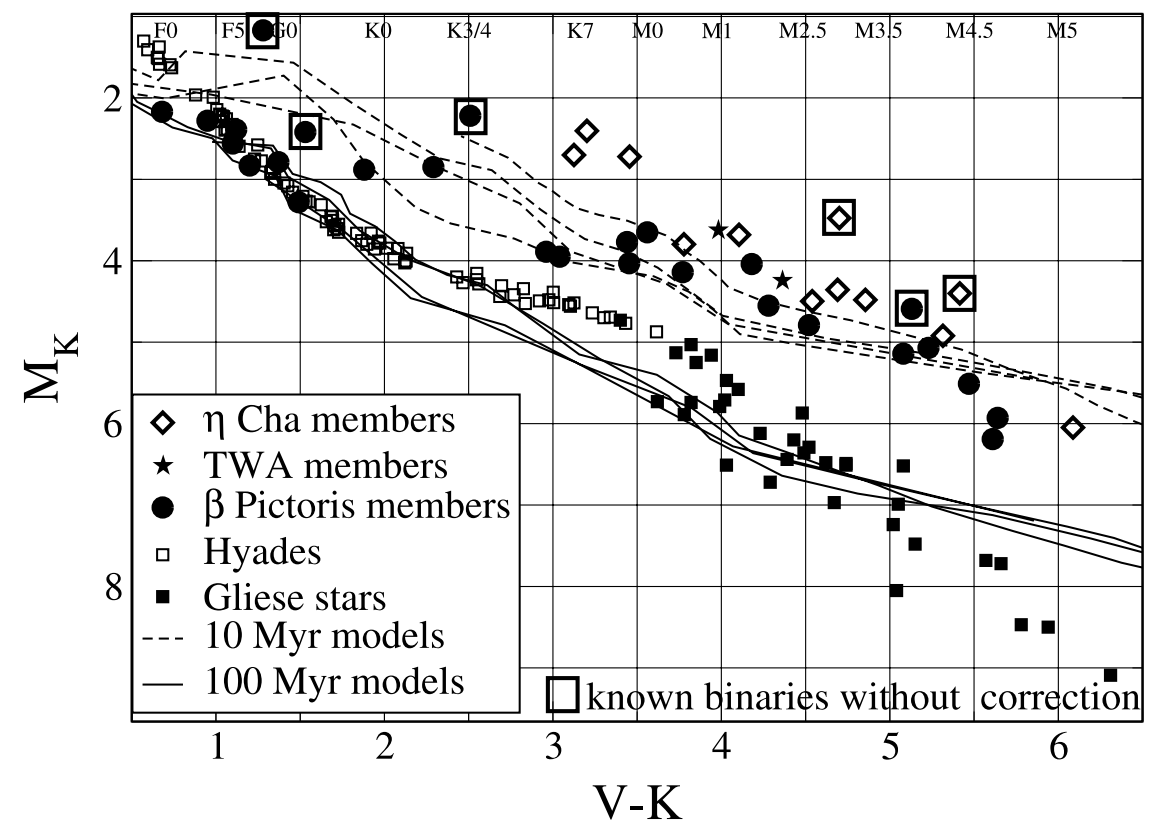

FIG. 2.-Absolute $K$ magnitude versus $V-K$ color of main-sequence and pre-main-sequence stars. All plotted stars have Hipparcos measured distances. For a reliability check of theoretical evolutionary tracks, members of $\eta$ Cha (5-8 Myr old; Lawson \& Feigelson 2001) and TWA ( $\sim 8$ Myr old) are plotted. Isochrones from four solar-metallicity evolutionary models are plotted at 10 and $100 \mathrm{Myr}$ (Baraffe et al.1998; D'Antona \& Mazzitelli 1997; Palla \& Stahler 1993; Siess et al. 2000). To determine the location of old main-sequence stars, Hyades stars and late-type Gliese stars with well-measured distances are plotted. Plotted Hyades stars are "high-fidelity" members as defined in de Bruijne, Hoogerwerf, \& de Zeeuw (2001).

TABLE 2

Distances and Galactic $U V W$ Velocities of TWA Members

\begin{tabular}{|c|c|c|c|c|c|c|c|}
\hline Name & $\begin{array}{c}\text { Trig. } \\
\text { Distance } \\
\text { (pc) }\end{array}$ & $\begin{array}{c}\text { Photometric } \\
\text { Distance } \\
\text { (pc) }\end{array}$ & $\begin{array}{c}\text { Radial } \\
\text { Velocity } \\
\left(\mathrm{km} \mathrm{s}^{-1}\right)\end{array}$ & $\begin{array}{l}U \text { Velocity } \\
\left(\mathrm{km} \mathrm{s}^{-1}\right)\end{array}$ & $\begin{array}{c}V \text { Velocity } \\
\left(\mathrm{km} \mathrm{s}^{-1}\right)\end{array}$ & $\begin{array}{c}W \text { Velocity } \\
\left(\mathrm{km} \mathrm{s}^{-1}\right)\end{array}$ & $\begin{array}{c}\text { Radial Velocity } \\
\text { Reference }\end{array}$ \\
\hline TWA 1 & 57 & 63 & 12.7 & -11.9 & -18.1 & -5.0 & 1 \\
\hline TWA 2 & $\ldots$ & 52 & 11.0 & -15.3 & -18.8 & -7.4 & 1 \\
\hline TWA 3A & $\ldots$ & 42 & 13.4 & -13.3 & -19.5 & -4.9 & 2 \\
\hline TWA 4 & 47 & 40 & 12.8 & -9.5 & -19.2 & -3.0 & 2 \\
\hline 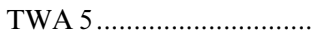 & $\ldots$ & 50 & 9.5 & -11.7 & -17.9 & -7.6 & 2 \\
\hline TWA $6 \ldots \ldots \ldots \ldots \ldots \ldots$ & $\ldots$ & 77 & 16.9 & -12.9 & -22.1 & -11.0 & 2 \\
\hline 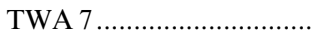 & $\ldots$ & 38 & 11.8 & -14.9 & -18.1 & -10.1 & 1 \\
\hline TWA 8A & $\ldots$ & 21 & 7.8 & $\ldots$ & $\ldots$ & $\ldots$ & 2 \\
\hline 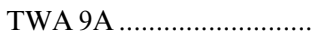 & 50 & 81 & 9.5 & -12.3 & -18.6 & -6.6 & 1 \\
\hline 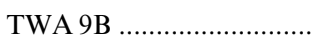 & 50 & 74 & 11.3 & $\ldots$ & $\ldots$ & $\ldots$ & 2 \\
\hline 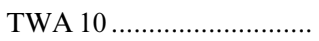 & $\ldots$ & 57 & 6.6 & -10.5 & -17.9 & -8.2 & 2 \\
\hline 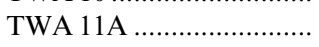 & 67 & 71 & 6.3 & -10.4 & -16.6 & -5.5 & 1 \\
\hline 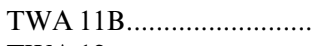 & 67 & 65 & $\ldots$ & $\ldots$ & $\ldots$ & $\ldots$ & . \\
\hline 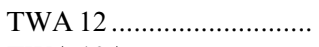 & $\ldots$ & 32 & 10.9 & $\ldots$ & $\ldots$ & $\ldots$ & 2 \\
\hline 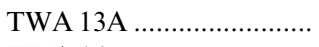 & $\ldots$ & 38 & 11.7 & $\ldots$ & $\ldots$ & $\ldots$ & 1 \\
\hline TWA 14 & $\ldots$ & 46 & 16.0 & $\ldots$ & $\ldots$ & $\ldots$ & 2 \\
\hline 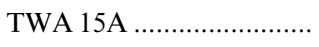 & $\ldots$ & 119 & 11.2 & $\ldots$ & $\ldots$ & $\ldots$ & 2 \\
\hline TWA $16 \ldots \ldots \ldots \ldots \ldots$ & $\ldots$ & 66 & 9.0 & $\ldots$ & $\ldots$ & $\ldots$ & 2 \\
\hline 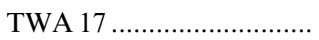 & $\ldots$ & 133 & 4.6 & $\ldots$ & $\ldots$ & $\ldots$ & 2 \\
\hline 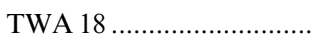 & $\ldots$ & 98 & 6.9 & $\ldots$ & $\ldots$ & $\ldots$ & 2 \\
\hline 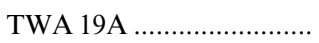 & 104 & 84 & 14.7 & -7.5 & -20.7 & -5.8 & 2 \\
\hline Average $U V W \ldots \ldots \ldots \ldots \ldots$ & & & & -11.4 & -18.8 & -6.5 & \\
\hline
\end{tabular}

Notes.- $U V W$ velocities are calculated for stars with good proper-motion data (i.e., Hipparcos or Tycho stars). Except for TWA 9A, for which the photometric distance of $81 \mathrm{pc}$ was used, trigonometric distances were used in the calculation of $U V W$ when they were available. Radial velocities are from (1) Torres et al.2003 and (2) Reid 2003. Average $U V W$ are calculated, including three newly identified Tycho members from Table 1 also. We believe that TWA 20 from Reid 2003 is not a TWA member. Although it is a young star $(<\sim 30$ Myr), its $\mathrm{Li} \lambda 6708$ equivalent width $(160 \mathrm{~mA})$ is too small to be consistent with TWA membership (see I. Song et al. 2004, in preparation). 
$\mu_{\delta}=-40$ mas $\mathrm{yr}^{-1}$ ). The combination of youth, proximity to Earth, and very late spectral type suggests that this star, if single, could be one of the best in the sky for an imaging search for a cooling planet.

HIP 23418 has a faint $\left(\Delta m_{v} \sim 1 \mathrm{mag}\right)$ companion about 0 ".85 southwest of the primary (see Hipparcos doubles and multiples catalog for details). We have corrected the absolute $K$ magnitude plotted in Figure 2 to take into account the light contribution from the secondary. HIP 11437 is also a binary system, and we confirm common proper motions of the two components from POSS-I (epoch 1954) and POSS-II (epoch 1989) plate positions.

The HIP 112312 (WW PsA) binary system is particularly interesting. The primary $(\sim \mathrm{M} 4)$ and secondary $(\sim \mathrm{M} 4.5)$ differ in spectral type by only a half-subclass, but the primary does not show any Li $\lambda 6708$ feature, while the secondary shows a strong Li absorption feature. The detection and nondetection of $\mathrm{Li}$ in the HIP 112312 binary is interpreted as a "lithium depletion boundary" (LDB) in a $\sim 12$ Myr old stellar group. This unusual binary can be used as a strong observational constraint to calibrate pre-main-sequence evolutionary tracks. Details have been reported in a separate paper (Song, Bessell, \& Zuckerman 2002b).

The observed LDB in the $\beta$ Pic group is corroborated with detection of a strong Li $\lambda 6708$ feature (Table 1) in an $\sim$ M4.5 companion $\sim 10^{\prime \prime}$ northwest of a previously known member, V343 Nor (=HIP 76629). A high surface density of young background stars, the Upper Centaurus-Lupus region (UCL; one of the three subgroups of the ScorpioCentaurus [Sco-Cen] association with an age similar to that of the $\beta$ Pic group), exists toward V343 Nor. Therefore, proximity of V343 Nor B to V343 Nor in the projected sky plane and strong Li $\lambda 6708$ detection alone cannot confirm the physical companionship of the two stars. To check the reality of the binary nature of V343 Nor, we obtained $V I_{C}$ magnitudes with the $0.9 \mathrm{~m}$ telescope at SSO. These photometric data clearly reject the possibility of V343 Nor B being a UCL member $\sim 140$ pc from Earth. Furthermore, common proper motions calculated from the UK Schmidt Telescope blue plate positions (epoch 1975) and our measured CCD image positions (epoch 2002) confirm the physical companionship of V343 Nor B and V343 Nor A.

HIP 23418 and HIP 112312 A show prominent emission features of $\mathrm{Na} \mathrm{D} \lambda 5890$ and $\lambda 5896$, He I $\lambda 5876$ and $\lambda 6678$, and $\mathrm{H} \alpha$ (Fig. 3). HIP $112312 \mathrm{~B}$ also shows a hint of such emission features; however, signal-to-noise ratios of the spectra are too low to draw any definitive conclusions. We note that, along with V343 Nor B, HIP 23418 and 112312 A and $\mathrm{B}$ are the latest-spectral type stars identified in the $\beta$ Pic group. Earlier-spectral type $\beta$ Pic members do not show these emission features. While $\mathrm{Na}$ and $\mathrm{He}$ emission features are prevalent among $\mathrm{T}$ Tauri stars (Beristain, Edwards, \& Kwan 2001), at the age of the TWA ( $8 \mathrm{Myr})$, these emission features are present only among late-type stars. Therefore, we believe that at $\sim 10 \mathrm{Myr}$, these emission features are present only among spectral types later than M3-4. It will be interesting to see if the same emission features persist to the Tucana/HorA age ( $30 \mathrm{Myr})$; however, no such late-type Tucana/HorA member has yet been identified.

All $\beta$ Pic moving group members identified by Zuckerman et al. (2001a) lie in the southern hemisphere, while most of the newly identified systems are in the northern hemisphere (Table 1). These additional new members now

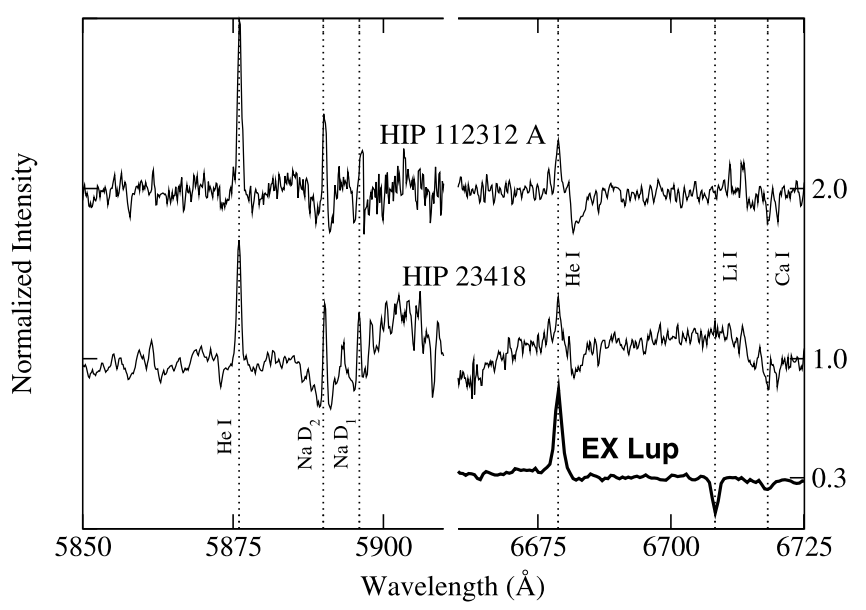

FIG. 3.-Emission features other than $\mathrm{H} \alpha$ seen at HIP 23418 and HIP 112312 A. Similar emission features are present in the spectra of HIP $112312 \mathrm{~B}$, but because of the low signal-to-noise ratios, they are not plotted. A DBS spectrum of the well-known T Tauri star, EX Lup (M0 spectral type), is plotted as a dark solid line for comparison.

indicate the $\beta$ Pic group to be dispersed up to $\sim 100 \mathrm{pc}$ along its long dimension. To check if such widely separated stars share the same birthplace, we traced the positions of Table 1 and Zuckerman et al. (2001a) $\beta$ Pic group members back in time (Fig. 4). In this backward time integration, we

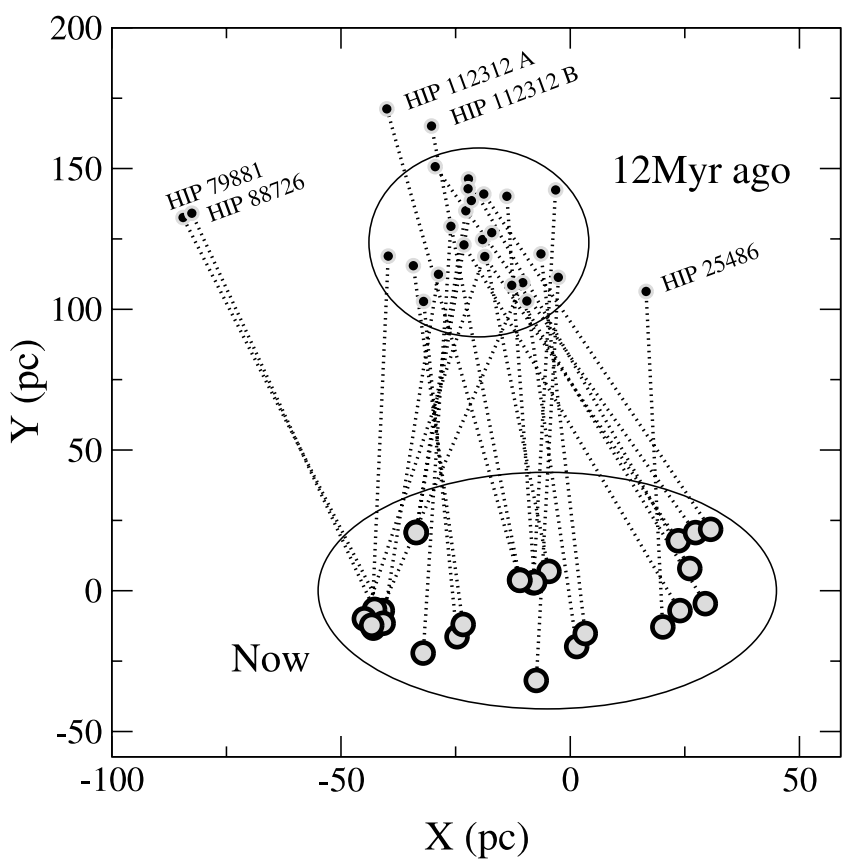

FIG. 4.-Positions of $\beta$ Pic moving group members back-traced $12 \mathrm{Myr}$ in time. $X$ and $Y$ are distances in the Cartesian corotating local standard of rest (LSR) frame, where $X$ is positive toward the Galactic anticenter and $Y$ is positive in the direction of the Galactic rotation. We used $U V W$ values $(-10.00,-5.23,-7.17)$ for the velocity of the Sun with respect to the LSR in kilometers per second, adapted from Dehnen \& Binney (1998). HIP 12545 is a radial velocity variable star, and we have used an estimated system radial velocity of $+10.0 \mathrm{~km} \mathrm{~s}^{-1}$. HIP 95261 has multiple radial velocity measurements, with large uncertainties in the range of -17 to +13 , and we used $+0.0 \mathrm{~km} \mathrm{~s}^{-1}$ for its radial velocity in our calculation. HIP 79881 and HIP 88726 are A-type stars listed in Table 1 of Zuckerman et al. (2001a), and their radial velocity uncertainties are large $\left(\gtrsim 10 \mathrm{~km} \mathrm{~s}^{-1}\right)$. 
calculated stellar positions using constant velocity trajectories, i.e., no influence of Galactic potential. Excepting a few outliers (HIP 79881, HIP 88726, and HIP 25486), all members were confined in a smaller space about $\sim 12$ Myr ago. This is in a good agreement with the result of Ortega et al. (2002), who found that $\beta$ Pic members from Zuckerman et al. (2001a) occupied the smallest volume about $11.5 \mathrm{Myr}$ ago by using a realistic Galactic potential field.

Backward time integration is quite sensitive to input data; travel distance with velocity of $1 \mathrm{~km} \mathrm{~s}^{-1}$ during $1 \mathrm{Myr}$ is $\sim 1$ pc. This is the main reason why backward time integration is applicable only to very young ( $\lesssim 15 \mathrm{Myr}$ ) stars or stars with large space velocity (see, for example, Hoogerwerf, de Bruijne, \& de Zeeuw 2000). For 30 Myr old stars (e.g., Tucana/HorA) with typical space velocity uncertainty of 2 $\mathrm{km} \mathrm{s}^{-1}$, uncertainties in back-traced positions would be $\sim 60$ $\mathrm{pc}$, which is too large to extract any reasonable birthplace information. For the $\beta$ Pic members, back-traced positions $12 \mathrm{Myr}$ ago have uncertainties of $\sim 25 \mathrm{pc}$. Two binary systems among the newly identified $\beta$ Pic members (HIP 10680/HIP 10679 and HIP 112312 A and B) can be used to estimate errors of a back-tracing calculation more rigorously. HIP $112312 \mathrm{~A}$ and B are currently separated by $\sim 36^{\prime \prime}$ (850 AU) in the projected sky plane; however, back-traced positions $12 \mathrm{Myr}$ ago are separated by $11.5 \mathrm{pc}$, which is physically implausible. At their birth, gravitationally bound systems like HIP 112312 must not have been separated significantly more than their separation now; hence, we regard the discrepancy of $11.5 \mathrm{pc}$ as indicative of the uncertainty in this type of calculation. Similarly, HIP 10680 and HIP 10679 are currently separated by $\sim 14^{\prime \prime}$ in the projected sky plane, and each has a Hipparcos parallax measurement (HIP 10679: $\pi=29.40 \pm 5.39$ mas; HIP 10680: $\pi=$ $25.37 \pm 2.84$ mas). Given these error bars, and the fact that HIP 10679 and 10680 are very close together in the plane of the sky and share the same proper motions and radial velocity, we believe that the two stars are in fact located at the same distance from Earth. At the weighted mean distance of $38.1 \pm 3.6 \mathrm{pc}$, the two stars are currently separated by $\sim 530$ AU, but they were separated by $39 \mathrm{pc}$ in the back-traced positions $12 \mathrm{Myr}$ ago; again, this is physically unrealistic. Such uncertainties are direct consequences of uncertainties in various input parameters, such as radial velocities, proper motions, and distances.

Although we cannot trust too much an individual star's back-traced position, as an ensemble of stars, the $\beta$ Pic group clearly occupied a smaller volume $\sim 12 \mathrm{Myr}$ ago, and this dynamic age is in good agreement with the photometric isochrone age (Fig. 1 of Zuckerman et al. 2001a and Fig. 2 of this paper). Considering the uncertainty of the backtraced positions, we cannot distinguish between two formation scenarios: (1) the size of the $\beta$ Pic group at the time of its formation was $\sim 30 \mathrm{pc}$, or (2) the $\beta$ Pic group was formed in a very compact region (a few parsecs) but with a large velocity dispersion. For the former case, the initial size of the $\beta$ Pic group is comparable to that of the entire Taurus complex, which is about $40 \mathrm{pc}$, not including the nearby Auriga region. This picture of the $\beta$ Pic group formation is very plausible if the "rapid star formation" model (e.g., Hartmann, Ballesteros-Paredes, \& Bergin 2001 and references therein) is the general mode of star formation. In this scenario, large-scale flows in the diffuse interstellar medium rapidly formed filamentary molecular clouds in which a small part ( $\sim 50 \mathrm{pc})$ of the clouds (a few hundred parsecs in size) eventually gave birth to the $\beta$ Pic group. For the latter scenario, the current extension of the $\beta$ Pic group members implies an initial velocity dispersion of $\sim 8 \mathrm{~km} \mathrm{~s}^{-1}$, which is about 4 times larger than the velocity dispersion in the Taurus molecular cloud (Kleiner \& Dickman 1985). It is interesting to note that the velocity dispersion of coeval groups of stars in the solar neighborhood is thought to be $\sim 8 \mathrm{~km} \mathrm{~s}^{-1}$ at birth (Binney, Dehnen, \& Bertelli 2000).

\subsection{New Members of the TW Hydrae Association}

Unlike the $\beta$ Pic and Tucana/HorA groups, which contain a wide range of spectral types, the $\sim 8 \mathrm{Myr}$ old TWA is dominated by late $\mathrm{K}$ - and M-type stars. A unique aspect of these late-type TWA members is a strong ( 400 $\mathrm{mA})$ and ubiquitous Li $\lambda 6708$ feature (Fig. 5). Already by the age of $\sim 12 \mathrm{Myr}$, characteristic of the $\beta$ Pic moving group, the Li $\lambda 6708$ line is either much weaker or not detected among Mtype stars. As shown in Table 1, all new TWA members show a strong Li $\lambda 6708$ feature, which indicates their very young age. Also, all the new TWA members show $\mathrm{H} \alpha$ either in emission or filled in. With a TWA age of $\sim 8 \mathrm{Myr}$, photometric distances of Table 1 TWA members are estimated from our $B V I_{\mathrm{C}}$ measurements and 2MASS $J H K_{s}$ photometry.

The TWA is located close to the Sco-Cen complex, which is the nearest recent massive star formation site to Earth. The Sco-Cen complex consists of three subgroups, which may be distinguished by different locations, space motions, and ages (see de Zeeuw et al. 1999 for details). The Lower Centaurus-Crux (LCC) region, the closest $(\sim 120 \mathrm{pc})$ to Earth among the three subregions, is very similar to the TWA in various ways. The age of the LCC is thought to be

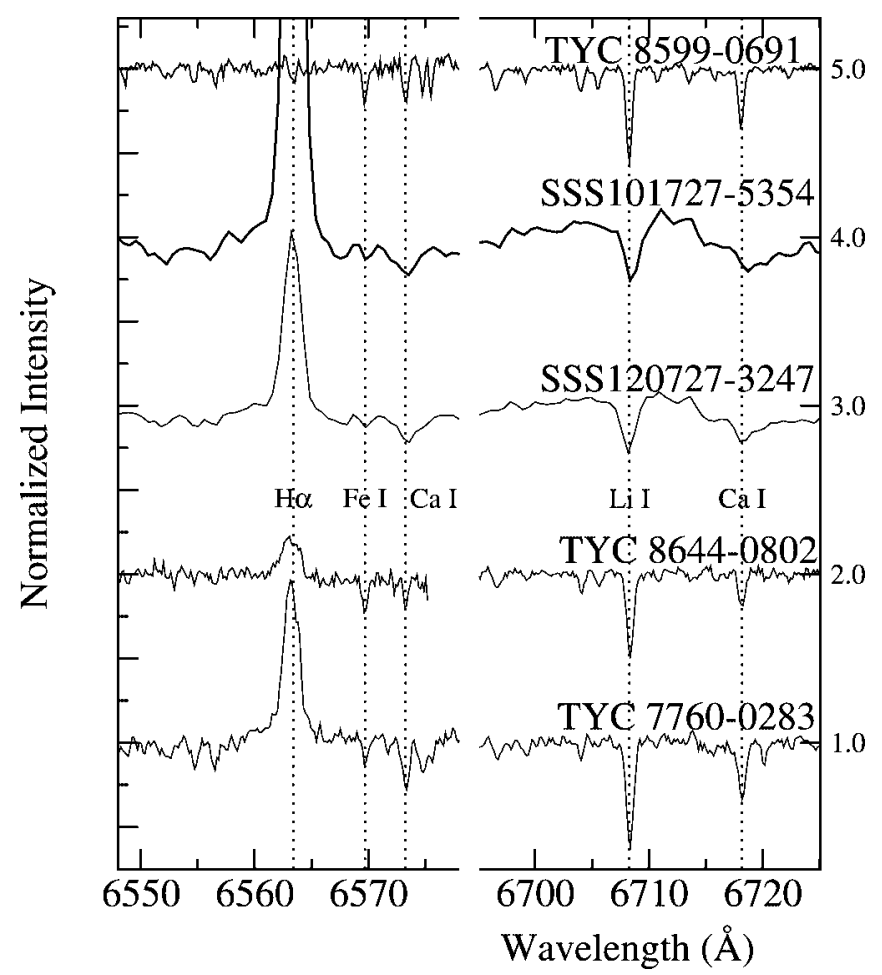

FIG. 5.-Echelle spectra of three newly identified TWA members from the Tycho catalog and DBS spectra of SSS 101727-5354 and SSS 120727-3247 from the SuperCOSMOS catalog. 


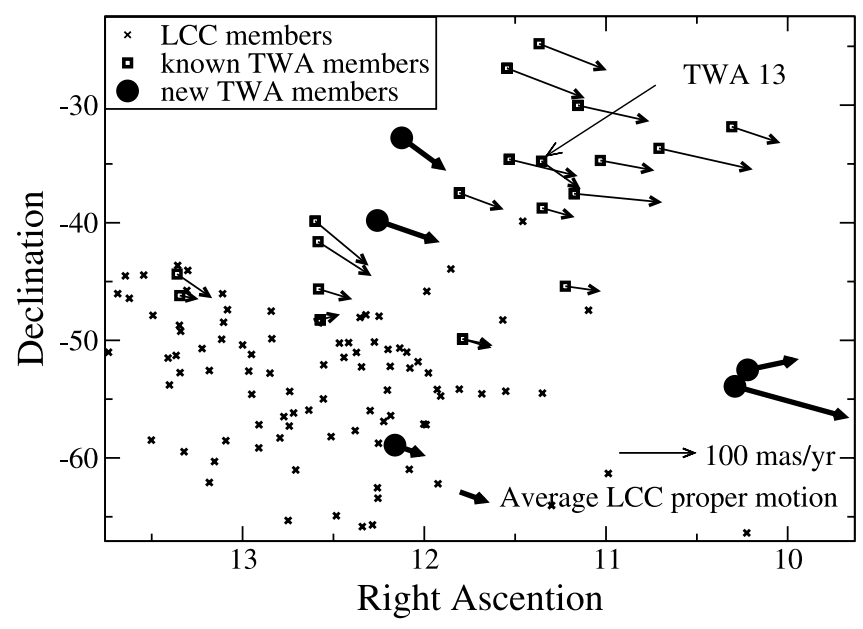

FIG. 6.-Positions and proper motions of TWA and LCC members in the projected sky plane. LCC membership is based on de Zeeuw et al. (1999), and proper motions of TWA members are from the Hipparcos, Tycho-2, UCAC-1, or SuperCOSMOS catalog. TWA 13 has proper motions incorrectly listed in the UCAC- 1 catalog $\left(\mu_{\alpha}=-14.0\right.$ mas $\mathrm{yr}^{-1}$ and $\mu_{\delta}=+41.9$ mas $\left.\mathrm{yr}^{-1}\right)$, possibly because of its close binary nature. We have estimated proper motions of TWA $13\left(\mu_{\alpha}=-48{\text { mas } \mathrm{yr}^{-1} \text { and }}^{-1}\right.$

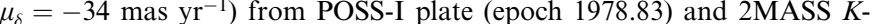
band image (epoch 1999.31) positions. Uncertainties in our measurement are likely to be large $\left(\sim 30\right.$ mas $\left.\mathrm{yr}^{-1}\right)$ because of the crude pixel scale of the POSS-I image. Arrows indicate magnitude and direction of proper motions.

$10-15 \mathrm{Myr}$, and its location in the projected sky plane is quite close to the TWA (the TWA and the northwest corner of the LCC overlap each other; see Fig. 6). Galactic motion $(U, V, W=-9,-18,-6)$ of stars in the northwest corner of the LCC is very similar to that in the TWA $(-11,-18$, -5 ). Therefore, the TWA may well be a subregion of the LCC group.

Makarov \& Fabricius (2001) argue that the TWA is a part of the Sco-Cen complex and that the Sco-Cen complex is, in turn, part of an even bigger structure, the "Gould Disk." However, the claim made by Makarov \& Fabricius (2001) was based on an analysis including kinematically selected candidate TWA members, most of which turned out to be nonmembers from spectroscopic follow-up observation (Song, Bessell, \& Zuckerman 2002a). Therefore, it is not yet clear if the TWA is indeed a part of the bigger LCC group. More thorough identification of members in the Sco-Cen complex is necessary to answer such a question.

Because of this ambiguity of membership between the TWA and LCC, we list young stars only within $\sim 100 \mathrm{pc}$ of Earth as possible TWA members. For example, our DBS spectra indicate that there are some quite young stars located farther away than $100 \mathrm{pc}$ in the TWA region; these are SSS 111028-3731, SSS 112105-3845, SSS 113218-3019, and SSS 123504-4136.

Following the same procedure for the distance estimation of TYC 8599-0691 in $\S 2.1$, distances of all TWA stars (new and previous members) are calculated by placing stars on the observational $10 \mathrm{Myr}$ isochrone in Figure 2. With these distances and radial velocities from the literature, we calculated Galactic velocities of TWA members with proper motion measurements, i.e., Hipparcos or Tycho stars (Table 2). As noted in Frink (2001), TWA members seem to have a longer dimension along the line of sight $(\sim 80 \mathrm{pc})$ than on the projected sky plane. If true, the TWA is a finger-like structure stretching out from the LCC region toward Earth. If not, it indicates that more TWA members remain to be found farther out from the apparent center of the association.

\subsection{New Tucana/HorA Members}

Previously known members of Tucana/HorA are confined mostly in the right ascension (R.A.) range $19^{\mathrm{h}}-2^{\mathrm{h}}$. In this study, we have found 11 new Tucana/HorA members in the R.A. range $2^{\mathrm{h}}-7^{\mathrm{h}}$ (Table 1). These new members have slightly different Galactic space motions $(\Delta U \sim 4$ and $\Delta W \sim 3 \mathrm{~km} \mathrm{~s}^{-1}$ ) compared to the $U V W$ of the members in the Tucana nucleus (see $\S 3$ of Zuckerman \& Webb 2000). The same trend is seen in the newly identified $\beta$ Pic members- $U$ velocities more negative than those identified by Zuckerman et al. (2001a). Because new members are found in a different part of the sky, in order to have the same birthplace, members of these nearby young groups need to have slightly different $U V W$ velocities, depending on their positions in the projected sky plane.

All newly identified Tucana/HorA members share the same space motion and smiliar distance from Earth (50$60 \mathrm{pc}$ ) and have Li $\lambda 6708$ line strengths comparable to that of IC 2602 ( $\sim 30$ Myr old; Figs. 7 and 8). $\mathrm{H} \alpha$ absorption lines are all filled in, compared to $\mathrm{H} \alpha$ equivalent widths of the same spectral type stars in Panagi \& Mathioudakis (1993). Relevant observed data are provided in Table 1.

During our ongoing spectroscopy program, we obtained spectra of suggested Tucana/HorA members from Zuckerman et al. (2001b) that lacked spectroscopic data.

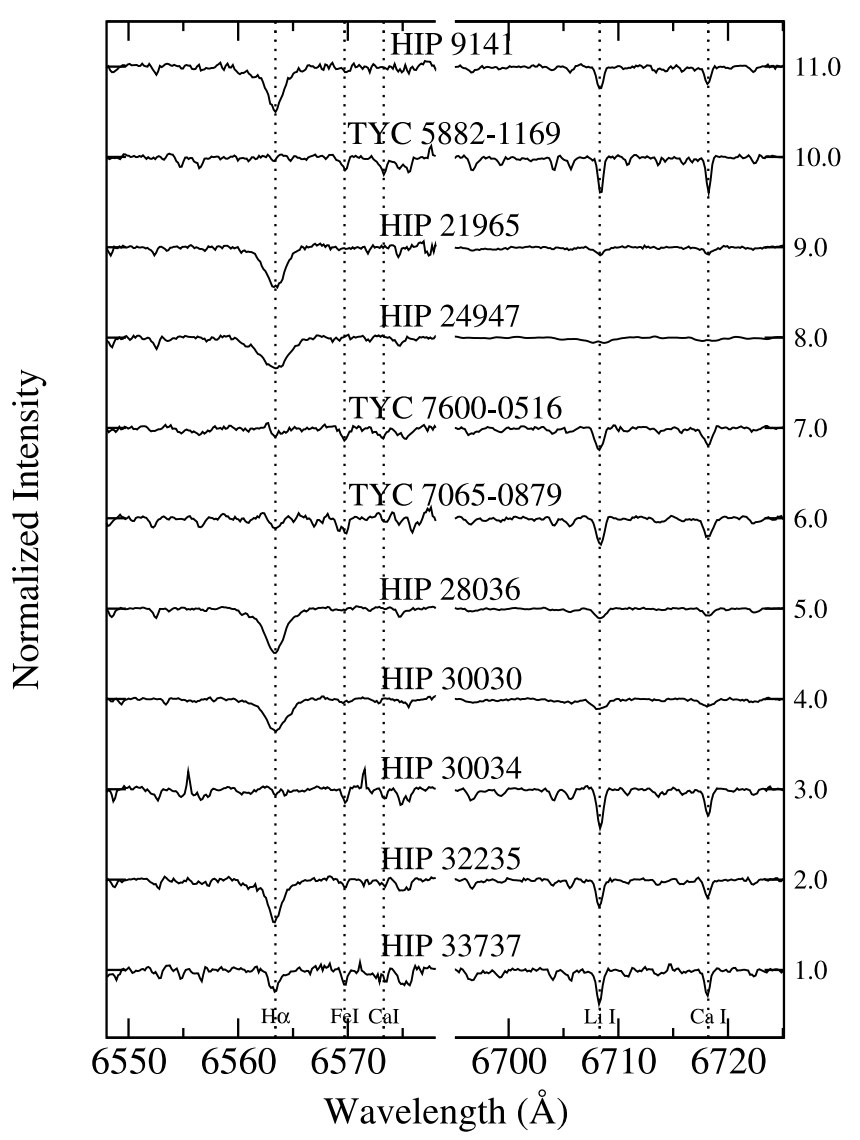

FIG. 7.-Echelle spectra of newly identified Tucana/HorA members 


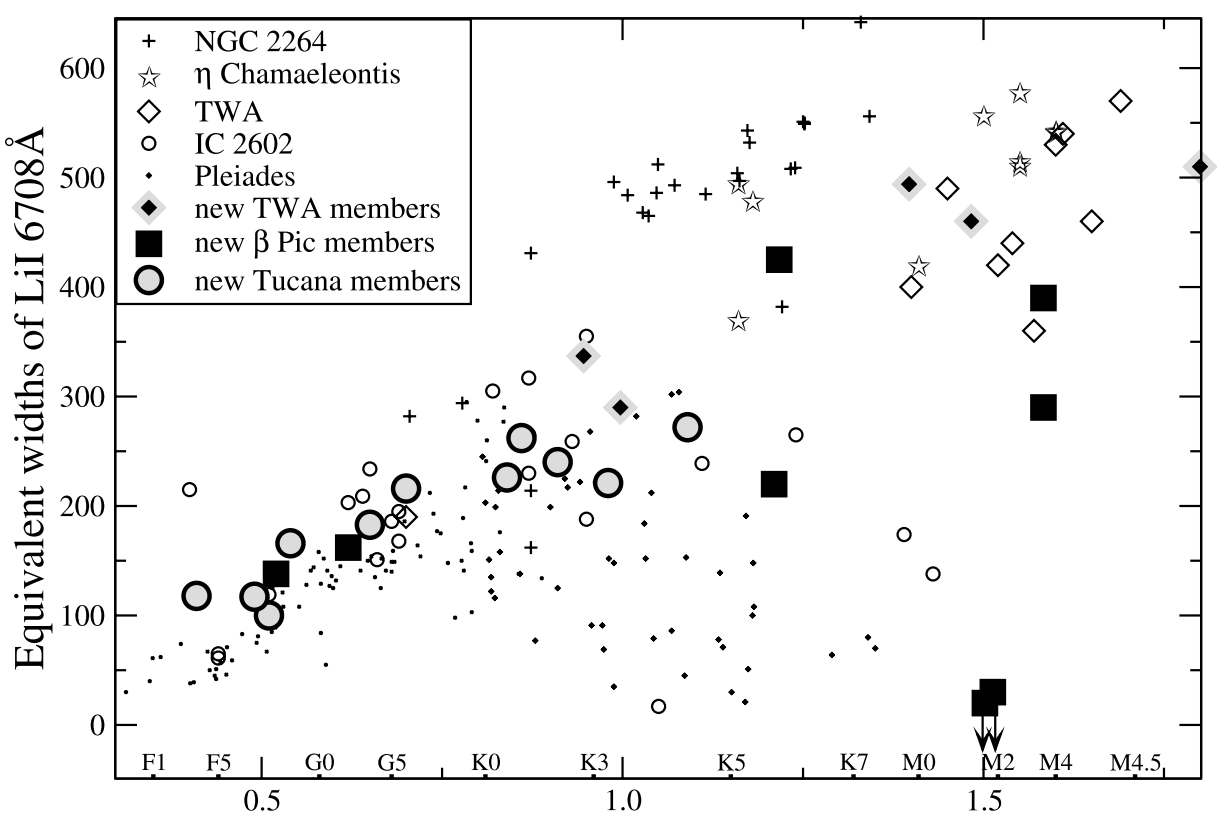

$(\mathrm{B}-\mathrm{V})$

Fig. 8. - Equivalent width of $\mathrm{Li} \lambda 6707.76$ as a function of $B-V$. Displayed equivalent widths are not corrected for possible contamination by Fe $\mathrm{I} \lambda 6707.44$, and measurement uncertainty of equivalent widths is about $20 \mathrm{~m} \AA$. Plus signs indicate NGC 2264 stars ( $<5 \mathrm{Myr})$, small open circles denote IC 2602 stars ( $\sim 30 \mathrm{Myr}$ ), and dots represent Pleiades stars ( $<100 \mathrm{Myr}$; see Song et al. 2002b for IC 2602 and Pleiades age). Previously known TWA members are plotted as open diamonds. New TWA, $\beta$ Pic, and Tucana/HorA members are displayed as large filled diamonds, squares, and circles, respectively.

From these spectra, we now know that HD 5578, HD 186602, HD 202746, and HD 207129 either have too weak a $\mathrm{Li} \lambda 6708$ feature or inappropriate $U V W$ values to be Tucana/HorA members. In Zuckerman \& Webb (2000), reasons were provided as to why the G0 star HD 207129 was more likely to be a $\sim 30$ Myr old Tucana member than a 4.7 Gyr old star, as suggested elsewhere. Those reasons were (1) X-ray luminosity 10 times greater than that of the Sun,

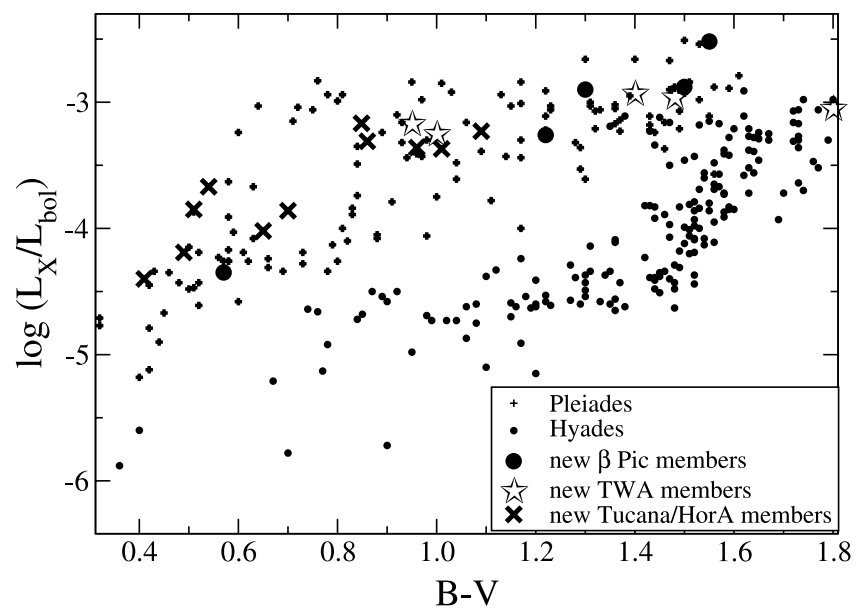

FIG. 9.-Fractional X-ray luminosities of newly identified members of the TWA, $\beta$ Pic, and Tucana/HorA groups. For comparison, Pleiades $(<100 \mathrm{Myr}$ old) and Hyades ( $600 \mathrm{Myr}$ old) members are plotted. Young $\mathrm{K}$ and $\mathrm{M}$ stars are believed to have saturated $\mathrm{X}$-ray activities, $L_{\mathrm{X}} / L_{\text {bol }} \sim 10^{-3}$, which are clearly seen among the new group members. Excepting V343 Nor B, each $\beta$ Pic binary in Table 1 has been plotted as a single point.
(2) $U V W$ velocity very similar to that of Tucana members, along with a small probability of having such velocity by chance, and (3) excess IR emission, which is seen more frequently in young stars. While the above arguments remain true, our measured Li $\lambda 6707.76$ line equivalent width of HD 207129 (42 $\mathrm{m} \AA$; without Fe I $\lambda 6707.44$ correction) is too small for HD 207129 to be a Tucana member (Fig. 8). However, this Li equivalent width also supports the contention that HD 207129 is not $4.7 \mathrm{Gyr}$ old on the basis of the $\mathrm{Li}$ $\lambda 6708$ equivalent width distribution of Hyades solar-type

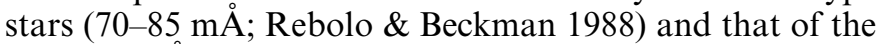
Sun ( $<6 \mathrm{~mA}$; Brault \& Mueller 1975). The fractional X-ray luminosity of HD 207129, $\log L_{\mathrm{X}} / L_{\mathrm{bol}}=-5.66$, is comparable to those of Hyades stars with similar $B-V$ $(=0.60$; Fig. 9), while the fractional far-IR luminosity $L_{\mathrm{IR}} / L_{\mathrm{bol}}=6.5 \times 10^{-5}$ (Silverstone 2000 ) is relatively large for a dusty debris disk of Hyades age (see Fig. 1 in Spangler et al. 2001). On balance, we consider HD 207129 to be probably about as old as the Hyades.

\section{DISCUSSION AND SUMMARY}

X-ray-to-bolometric flux ratios, $\log L_{\mathrm{X}} / L_{\mathrm{bol}}$, are calculated for all identified new members of the three kinematic groups. All stars with $B-V$ larger than 0.8 ( $\mathrm{K}$ and $\mathrm{M}$ stars) show saturated X-ray activities, $L_{\mathrm{X}} / L_{\mathrm{bol}} \sim 10^{-3}$, while F/G-type stars show moderately reduced X-ray activity (Fig. 9). The same pattern is seen in the $\sim 30$ Myr old open cluster IC 2602 (Randich et al. 1995) and the 20-40 Myr old NGC 2547 (Jeffries \& Tolley 1998). This pattern is interpreted as an effect of different spin-down timescales between $\mathrm{F} / \mathrm{G}$ and $\mathrm{K} / \mathrm{M}$ stars, such that later type stars maintain higher rotational speed (hence, stronger X-ray activity) for 
a longer period of time. X-ray activity of newly found members of the three nearby young groups is stronger or comparable to that of the Pleiades. Saturated X-ray activity among $\mathrm{K} / \mathrm{M}$ stars can be used as an additional criterion to identify $\lesssim 30$ Myr old late-type stars.

We have found five new members of the TW Hydrae association. The proximity of some TWA members to the LCC region in space supports the idea that the TWA is a foreground part of the LCC region; however, more thorough investigation is necessary to substantiate such a claim. At the moment, we regard very young stars $(<10 \mathrm{Myr})$ within $100 \mathrm{pc}$ of Earth in the general direction of TW Hya as members of the TWA.

We found 11 new members of the $\beta$ Pic moving group: five binaries and a companion of a previously known member. Strong Li $\lambda 6708$ absorption features were detected in two $\sim$ M4.5 members. Given that lithium is not detected among members of the $\beta$ Pic group with spectral types only slightly earlier than $\sim \mathrm{M} 4.5$, we can set the lithium depletion boundary of the $12 \mathrm{Myr}$ old $\beta$ Pic group at $\sim \mathrm{M} 4.5$ (Song et al. 2002b). From its current spatial extent of $\sim 100 \mathrm{pc}$ and positions, we can deduce that the $\beta$ Pic group may have formed in a diffuse environment $30-40 \mathrm{pc}$ in size or in a compact region of a few parsecs across with large initial velocity dispersion of $\sim 8 \mathrm{~km} \mathrm{~s}^{-1}$.

We have found 11 new likely members of the Tucana/ Horologium association, most of which are located farther north than previously identified members. Newly obtained spectra show that some candidate members suggested earlier in the literature are not real members of the Tucana/ HorA.
Age estimation of medium-mass stars ( $\mathrm{F} / \mathrm{G}$ stars) is difficult because they arrive at the zero-age main sequence quickly ( $\lesssim 10 \mathrm{Myr}$ ) and other age indicators (e.g., Li $\lambda 6708$ strength) are not decisive for this mass range. Via measurements of common Galactic space motion $(U V W)$, we often can place $\mathrm{F} / \mathrm{G}$-type stars in a coeval group containing $\mathrm{K} / \mathrm{M}$-type stars. Since accurate ages can be deduced for pre-main-sequence $\mathrm{K} / \mathrm{M}$-type stars from placement on a CMD (Fig. 2) or Li $\lambda 6708$ line strengths (Fig. 8), we then also know the ages of the comoving F/G-type stars.

The TWA was relatively easy to identify because of large apparent X-ray luminosity (Kastner et al. 1997) and notable lithium line strengths (Webb et al. 1999) of its low-mass members. In addition, while much more spread out in the sky than previously known clusters, the TWA is still moderately localized in the plane of the sky (e.g., Fig. 6). In contrast, members of the $\beta$ Pic moving group are found over most of the sky, and lithium abundances are not necessarily large. Because of the difficulty in identifying common characteristics of this remarkable group of nearby stars, its rather large membership is only now becoming apparent. That this is possible at all is a tribute to the all-sky surveys (ROSAT, Hipparcos, IRAS, and 2MASS) of the past few years.

This research was supported in part by the UCLA Astrobiology Institute and by a NASA grant to UCLA. We thank N. C. Hambly for providing a digital version of the SuperCOSMOS catalog. This research has made use of the SIMBAD \& Vizier databases, operated at CDS, Strasbourg, France.

\section{REFERENCES}

Baraffe, I., Chabrier, G., Allard, F., \& Hauschildt, P. H. 1998, A\&A, 337, 403

Barrado y Navascués, D., Stauffer, J. R., Song, I., \& Caillault, J.-P. 1999, ApJ, 520, L123

Beristain, G., Edwards, S., \& Kwan, J. 2001, ApJ, 551, 1037

Binney, J., Dehnen, W., \& Bertelli, G. 2000, MNRAS, 318, 658

Brault, J. W. \& Mueller, E. A. 1975, Sol. Phys., 41, 43

Cohen, J. G., Persson, S. E., \& Frogel, J. A. 1978, ApJ, 222, 165

D’Antona, R. A., \& Mazzitelli, I. 1997, Mem. Soc. Astron. Italiana, 68, 807

de Bruijne, J. H. J., Hoogerwerf, R., \& de Zeeuw, P. T. 2001, A\&A, 367, 111

de La Reza, R., Torres, C. A. O., Quast, G., Castilho, B. V., \& Vieira, G. L. 1989, ApJ, 343, L61

de Zeeuw, P. T., Hoogerwerf, R., de Bruijne, J. H. J., Brown, A. G. A., \& Blaauw, A. 1999, AJ, 117, 354

Dehnen, W., \& Binney, J. J. 1998, MNRAS, 298, 387

Duggan, M. B., \& Knutson, J. 1991, The Warrumbungle Volcano, Central New South Wales: An Outline of the Geology and Petrology of the Warrumbungle Volcano (Canberra: Bureau of Mineral Resources)

Frink, S. 2001, in ASP Conf. Ser. 244, Young Stars Near Earth: Progress and Prospects, ed. R. Jayawardhana \& T. Greene (San Francisco: ASP), 16

Hambly, N. C., et al. 2001, MNRAS, 326, 1279

Hartmann, L., Ballesteros-Paredes, J., \& Bergin, E. A. 2001, ApJ, 562, 852

Hoogerwerf, R., de Bruijne, J. H. J., \& de Zeeuw, P. T. 2000, ApJ, 544, L133

Jacobson, S. B. 2003, Science, 300, 1513

Jayawardhana, R., \& Greene, T., ed. 2001, ASP Conf. Ser. 244, Young

Stars Near Earth: Progress and Prospects (San Francisco: ASP)

Jeffries, R. D., \& Tolley, A. J. 1998, MNRAS, 300, 331

Kastner, J. H., Zuckerman, B., Weintraub, D. A., \& Forveille, T. 1997, Science, 277, 67

Kenyon, S. J., \& Hartmann, L. 1995, ApJS, 101, 117
Kleiner, S. C., \& Dickman, R. L. 1985, ApJ, 295, 466

Lawson, W., \& Feigelson, E. D. 2001, in ASP Conf. Ser. 243, From Darkness to Light: Origin and Evolution of Young Stellar Clusters, ed. T. Montmerle \& P. André (San Francisco: ASP), 591

Makarov, V. V., \& Fabricius, C. 2001, A\&A, 368, 866

Ortega, V. G., de la Reza, R., Jilinski, E., \& Bazzanella, B. 2002, ApJ, 575, L75

Palla, F., \& Stahler, S. W. 1993, ApJ, 418, 414

Panagi, P. M., \& Mathioudakis, M. 1993, A\&AS, 100, 343

Randich, S., Schmitt, J. H. M. M., Prosser, C. F., \& Stauffer, J. R. 1995, A\&A, 300, 134

Rebolo, R., \& Beckman, J. E. 1988, A\&A, 201, 267

Reid, N. 2003, MNRAS, 342, 837

Siess, L., Dufour, E., \& Forestini, M. 2000, A\&A, 358, 593

Siess, L., Forestini, M., \& Dougados, C. 1997, A\&A, 324, 556

Silverstone, M. D. 2000, Ph.D. thesis, Univ. California, Los Angeles

Song, I., Bessell, M. S., \& Zuckerman, B. 2002a, A\&A, 385, 862

2002 b, ApJ, 581, L43

Spangler, C., Sargent, A. I., Silverstone, M. D., Becklin, E. E., \& Zuckerman, B. 2001, ApJ, 555, 932

Torres, C. A. O., da Silva, L., Quast, G. R., de la Reza, R., \& Jilinski, E. 2000, AJ, 120, 1410

Torres, G., Guenther, E. W., Marschall, L. A., Neuhäuser, R., Latham, D. W., \& Stefanik, R. P. 2003, AJ, 125, 825

Webb, R. A., Zuckerman, B., Platais, I., Patience, J., White, R. J., Schwartz, M. J., \& McCarthy, C. 1999, ApJ, 512, L63

Weis, E. W. 1993, AJ, 105, 1962

Zuckerman, B., Song, I., Bessell, M. S., \& Webb, R. A. 2001a, ApJ, 562, L87

Zuckerman, B., Song, I., \& Webb, R. A. 2001b, ApJ, 559, 388

Zuckerman, B., \& Webb, R. A. 2000, ApJ, 535, 959

Zuckerman, B., Webb, R. A., Schwartz, M., \& Becklin, E. E. 2001c, ApJ, 549, L233 